\title{
Phenylacetylglutamin und seine Bildung im menschlichen Körper nach Eingabe von Phenylessigsäure.
}

\author{
Von
}

H. Thierfelder und C. P. Sherwin. •

(Aus dem physiologisch-chemischen Institut der Universität Tübingen.)

(Der Redaktion zugegangen am 2. April 1915.)

Vor kurzem teilten wir $\left.^{1}\right)$ mit, daß nach Eingabe von Phenylessigsäure im Harn des Menschen nicht Phenacetursäure (wie bei Hunden und Kaninchen) oder Phenylacetornithursäure (wie bei Vögeln), sondern Phenylacetylglutamin erscheint und teils als Harnstoffverbindung teils als solches isoliert werden kann. Es gelang das Phenylacetylglutamin in die Phenylacetylglutaminsäure überzuführen und die Spaltungsprodukte dieser zu identifizieren. Wir haben uns inzwischen weiter mit diesen Substanzen beschäftigt mit folgenden Ergebnissen.

Die spezifische Drehung des Phenylacetylglutamin in 2 bis $40 \%$ iger wässeriger Lösung ist etwas höher $\left([\alpha]_{D}=-18^{\circ}\right)$, als wir angegeben. In salzsaurer Lösung nimmt die Drehung etwas zu. Die spezifische Drehung der Harnstoffverbindung in 3 bis $4 \%$ iger wässeriger Lösung ist $-14^{\circ}$. Die Phenylacetylglutaminsäure wurde auch krystallisiert erhalten, ihre spezifische Drehung beträgt in 3-10\% synthetische Phenylacetylglutaminsäure (aus d-Glutaminsäure) stimmt mit der natürlichen überein, auch in der spezifischen Drehung, und auch für das synthetische Phenylacetylglutamin (aus d-Glutamin) fanden wir völlige Übereinstimmung mit dem natürlichen. Von den Salzen krystallisiert das phenylacetylglutaminsaure Brucin gut, ebenso das Phenylacetylglutaminbaryum. Das Nähere siehe im experimentellen Teil.

4) Thierfelder und Sherwin, Chem. Ber., Bd. 47, S. 2630 (1914).

Hoppe-Serler'a Zeitechrift f. phrsiol. Chemie. XCIV. 
Das Auftreten der Glutaminverbindung im Harn zugunsten der Annahme, daß Glutamin ein normales Stoffwechselprodukt ist, zu verwerten lag nahe. Ein Beweis schien uns aber noch nicht erbracht, da Phenylacetylglutamin sekundär aus Phenylacetylglutaminsäure entstanden sein konnte. Wir haben inzwischen auf diesen Bildungsmodus experimentell geprüft. Der eine von uns (Sh.) nahm 4,5 g Phenylacetylglutaminsäure zu sich. Aus dem darauf gelassenen Harn ließ sich diese Säure wiedergewinnen, aber kein Phenylacetylglutamin, während das schon nach Einnahme von $1 \mathrm{~g}$ Phenylessigsäure mit Sicherheit gelang. Damit ist bewiesen, daß Glutamin im Stoffwechsel auftritt. Daß Glutamin und Asparagin an dem Aufbau des Eiweißmoleküls teilnehmen, ist schon von E. Fis cher ${ }^{1}$ ) angenommen und besonders von Th. B. Osborne ${ }^{2}$ ) wahrscheinlich gemacht worden. Osborne fand, daß Gliadin, ein Eiweißstoff, welcher bei der Hydrolyse sehr reichliche Mengen ( 43 bis $44 \%$ ) Glutaminsäure liefert, ${ }^{3}$ ) unter denselben Bedingungen Ammoniak abspaltet, unter denen auch aus dem Asparagin das amidartig gebundene Ammoniak frei wird und weiter, daß für eine große Anzahl von Eiweißstoffen die Mengen von Ammoniak und Glutaminsäure + Asparaginsäure, welche in dem Hydrolysat gefunden werden, ziemlich genau einander entsprechen in dem Sinne, daß auf 1 Mol. Ammoniak 1 Mol. Glutaminsäure oder Asparaginsäure kommt. Auf Grund dieser Feststellungen und der Ergebnisse unserer Untersuchung möchte man die Anwesenheit von Glutamin in dem Proteinmolekül für erwiesen halten. Indessen ist noch an die Möglichkeit zu denken, daß das Glutamin auch im tierischen Körper erst sekundär entsteht, wie in den Keimpflanzen das Asparagin, welches nach E. Schulze als ein sekundäres Produkt des Eiweißumsatzes auftritt.

Wir sind damit beschäftigt, zu versuchen, ob die Isolierung von Glutamin aus Liweiß gelingt und haben zu dem

1) E. Fischer und Königs, Chem. Ber., Bd. 37, S. 4585 (1904).

$\left.{ }^{2}\right)$ Osborne und Gilbert, Amer. Journ. of Physiol., Bd.15, S. 333 (1906), Osborne, Leavenworth und Brautlecht, Ebenda, Bd. 23, S. $184(1908 / 09)$.

3) Osborne und Guest, Jl. of Biol. Chem., Bd. 9, S. 425 (1911). 
Phenylacety]glutamin und seine Bildung im menschlichen Körper. 3

Zweck aus Gerstenmehl Hordein, welches nach den Untersuchungen von 0s borne bei der Spaltung mit Säure 43-44\% Glutaminsäure' liefert, dargestellt. Ist Glutamin im Eiweißmolekül vorgebịldet, so beweist das Auftreten von Phenylacetylglutamin im Harn, daß die polypeptidartige Bindung beim physiologischen Zerfall früher gelöst wird als die amidartige und es besteht die Hoffnung, daß es mit Hilfe geeigneter Fermente glücken wird, Glutamin abzuspalten.

Experimenteller Teil.

Phenylacetylglutamin.

Spezifische Drehung. Die beiden Bestimmungen wurden mit Präparaten verschiedener Darstellung in wässeriger Lösung ausgeführt.

1. 0,2384 g Substanz. Gesamtgewicht der Lösung 10,0058g. Spez. Gewicht 1,0066. Prozentgehalt 2,383. Drehung bei $17^{\circ}$ im $2 \mathrm{dm}-$ Rohr bei Natriumlicht $0,87^{\circ}$ nach links. Also $[\alpha]_{\mathrm{D}}^{17^{\circ}}$ $=-18,1^{\circ}$.

2. 0,2516 g Substanz. Gesamtgewicht der Lösung 7,3345 g. Spez. Gewicht 1,0042. Prozentgehalt 3,430. Drehung bei $18^{\circ}$ im $2 \mathrm{dm}$-Rohr bei Natriumlicht $1,27^{\circ}$ nach links. Also $[\alpha]_{D}^{18^{\circ}}$ $=-18,44^{\circ}$.

In salzsaurer Lösung (auf $1 \mathrm{Mol} .3 \mathrm{Mol} . \mathrm{HCl}$ ) ist die Drehung etwas größer:

$0,2113 \mathrm{~g}$ Substanz. Gesamtgewicht der Lösung $6,6530 \mathrm{~g}$. Spez. Gewicht 1,0190. Prozentgehalt 3,176. Drehung bei $18^{\circ}$ im $2 \mathrm{dm}$-Rohr bei Natriumlicht $1,27^{\circ}$ nach links. Also $[\alpha]_{\mathrm{D}}^{180}$ $=-19,62^{\circ}$.

Baryumsalz. Es schied sich aus der stark konzentrierten wässerigen Lösung zunächst an der Oberfläche $a b$; nach einiger Zeit erstarrte die ganze Flüssigkeit zu einer dicken Masse von kreideartigem Aussehen. Mikroskopisch Filzwerk von Nadeln.

$0,1950 \mathrm{~g}$ Substanz: $0,0590 \mathrm{~g} \mathrm{BaCO}_{3}=0,04106 \mathrm{~g} \mathrm{Ba}$

$\left(\mathrm{C}_{13} \mathrm{H}_{13} \mathrm{~N}_{2} \mathrm{O}_{4}\right)_{2}$ Ba ber. Ba 20,70

gef. "21,06. 
Die wässerige Lösung dreht rechts und zwar eine etwa $15 \%$ ige $0,25 \%$.

Synthese des Phenylacetylglutamins.

Das Glutamin war nach den Angaben von E. Schulze und Bosshard ') aus Runkelrübensaft dargestellt worden.

$0,1067 \mathrm{~g}$ Substanz: 0,1599 $\mathrm{g} \mathrm{CO}_{2}, 0,0671 \mathrm{~g} \mathrm{H}_{2} \mathrm{O}-0,1014 \mathrm{~g}$ Substanz: $17,4 \mathrm{ccm} \mathrm{N}\left(17^{\circ}, 741 \mathrm{~mm}\right)$.

$$
\begin{array}{r}
\mathrm{C}_{6} \mathrm{H}_{10} \mathrm{~N}_{2} \mathrm{O}_{3} \text { ber.: C } 41,07, \text { H } 6,90, \text { N } 19,18 \\
\text { gef.: > 40,87, > 7,05, >19,31. } \\
0,2061 \text { g Kupfersalz: } 0,0465 \mathrm{~g} \mathrm{CuO}=0,03715 \mathrm{~g} \mathrm{Cu} . \\
\left(\mathrm{C}_{6} \mathrm{H}_{9} \mathrm{~N}_{2} \mathrm{O}_{3}\right)_{2} \mathrm{Cu} \text { ber.: } \mathrm{Cu} 17,97 \\
\text { gef.: }>18,03 .
\end{array}
$$

Für die Polarisation dienten zwei Präparate verschiedener Darstellung.

Präparat a. 0,2539 g Substanz. Gesamtgewicht der Lösung $6,6851 \mathrm{~g}$. Spez. Gewicht 1,0154. Prozentgehalt 3,798. Drehung bei $14^{\circ}$ im $2 \mathrm{dm}$-Rohr bei Natriumlicht $0,503^{\circ}$ nach rechts. Also $[\alpha]_{\mathrm{D}}^{14^{\circ}}=+6,5^{\circ}$.

Präparat b. $0,2549 \mathrm{~g}$ Substanz. Gesamtgewicht der Lösung 6,7329 g. Spez. Gewicht 1,0148. Prozentgehalt 3,786. Drehung bei $14^{\circ} \mathrm{im} 2 \mathrm{dm}$-Rohr bei Natriumlicht $0,475^{\circ}$ nach rechts. Also $[\alpha]_{\mathrm{D}}^{14^{0}}=$ $+6,18^{\circ}$.

Nach den Bestimmungen von $E$. Schulze und Trier ${ }^{2}$ ) beträgt für das natürliche Glutamin in etwa $4 \%$ iger Lösung $[\alpha] \mathrm{D}+6^{\circ}$ bis $+7^{\circ}$.

Je $0,5 \mathrm{~g}$ Glutamin wurden mit $20 \mathrm{ccm}$ Wasser und $5 \mathrm{~g}$ Natriumbicarbonat unter allmählicher Zugabe von $3 \mathrm{~g}$ Phenylacetylchlorid eine halbe Stunde geschüttelt. Der beim Ansäuern auftretende Niederschlag wurde abgesaugt, das Filtrat mit Äther ausgeschüttelt und dann mehrere Stunden mit Äthylacetat extrahiert. Die Gewichte der nach dem Verdunsten des Äthylacetat sich ausscheidenden Krystallisationen schwankten zwischen 0,31 und $0,53 \mathrm{~g}$. Die Ausbeute entspricht also $34-60^{\circ} \%$ der Theorie.

Analyse. 0,1045 g Substanz: 0,2259 g CO $\mathrm{CO}_{2}, 0,0588 \mathrm{~g}$ $\mathrm{H}_{2} \mathrm{O}-0,1130 \mathrm{~g}$ Substanz: $10,9 \mathrm{ccm} \mathrm{N}\left(18^{\circ}, 739 \mathrm{~mm}\right)$

$$
\begin{array}{r}
\mathrm{C}_{13} \mathrm{H}_{16} \mathrm{~N}_{2} \mathrm{O}_{4} \text { ber.: } \mathrm{C} 59,06 \mathrm{H} 6,11 \mathrm{~N} 10,61 \\
\text { gef.: } * 58,96 \text { * } 6,29 \text { *10,71 }
\end{array}
$$

1) Landwirtsch. Versuchsstat., Bd. 29, S. 295 (1883); Chem. Ber., Bd. 16, S. 312 (1883).

2) Chem. Ber., Bd. 45, S. 257 (1912). 
Polarisation. 0,2214 g Substanz. Gesamtgewicht der Lösung 8,4119 g. Spez. Gewicht 1,0058. Prozentgehalt 2,632. Drehung bei $17^{\circ}$ im $2 \mathrm{dm}$-Rohr bei Natriumlicht $0,95^{\circ}$ nach links. Also $[\alpha]_{D}^{17^{\circ}}=-17,9^{\circ}$.

\section{Phen ylacetylglutaminharnst of .}

Spezifische Drehung. a) 0,2661 g Substanz. Gesamtgewicht der Lösung 8,0582 g. Spez. Gewicht 1,0081. Prozentgehalt 3,302. Drehung bei $15^{\circ} \mathrm{im} 2 \mathrm{dm}-$ Rohr $0,93^{\circ}$ nach links. Also $[\alpha]_{\mathrm{D}}^{15^{\circ}}=-13,97^{\circ}$.

b) 0,2440 g Substanz. Gesamtgewicht der Lösung 8,0367 g. Spez. Gewicht 1,0077 Prozentgehalt 3,036. Drehung bei $17^{\circ}$ im $2 \mathrm{dm}$-Rohr $0,874^{\circ}$ nach links. Also $[\alpha]_{\mathrm{D}}^{170}=-14,28^{\circ}$.

c) 0,2132 g Substanz. Gesamtgewicht der Lösung 6,5405 g. Spez. Gewicht 1,0079. Prozentgehalt 3,26. Drehung bei $18^{\circ}$ im $2 \mathrm{dm}$-Rohr $0,92^{\circ}$ nach links. Also $[\alpha]_{D}^{180}=-14,00^{\circ}$.

\section{Phenylacetylglutaminsäure.}

Wir erhielten sie, wie früher gesagt, durch Kochen von Phenylacetylglutamin mit gesättigtem Barytwasser. In dem Sirup, welcher nach Verdunsten ihrer essigätherischen Lösung zurückbleibt, beginnt nach kürzerer oder längerer Zeit eine Krystallisation, welche in blumenkohlartigen Gebilden über die Oberfläche hinauswächst. Schließlich ist alles in eine voluminöse Krystallmasse verwandelt. Auch aus Wasser krystallisiert sie. Nikroskopisch sternförmig gruppierte Nadeln. Bei $80^{\circ}$ im Vakuum getrocknet schmolz sie ziemlich scharf bei $123^{\circ}$.

Analyse. 0,1229 g Substanz: 0,2639 g CO$_{2}, 0,0632$ g $\mathrm{H}_{2} \mathrm{O}-0,2222 \mathrm{~g}$. Substanz: $11,18 \mathrm{ccm} \mathrm{N}\left(18^{\circ}, 713 \mathrm{~mm}\right)$.

$\mathrm{C}_{13} \mathrm{H}_{15} \mathrm{NO}_{5}$ ber. $\mathrm{C} 58,84 \mathrm{H} 5,70 \mathrm{~N} \mathrm{5,28}$

$$
\text { gef. }>58,56 \times 5,75 \times 5,42
$$

0,1061 g Substanz verbrauchen bei der Titration $7,9 \mathrm{ccm}$ n/10-Lauge statt der berechneten 8,00 .

Spezifische Drehung. 0,9969 g Substanz. Gesamtgewicht der Lösung $9,8333 \mathrm{~g}$. Spez. Gewicht 1,0269. Prozent- 
gehalt 10,138 . Drehung bei $18^{\circ} \mathrm{im} 2 \mathrm{dm}$-Rohr bei Natriumlicht $3,99^{\circ}$ nach links. Also $[\alpha]_{D}^{18^{\circ}}=-19,16^{\circ}$.

Baryumsalz. In der wässerigen Lösung bildete sich nach starker Konzentration auf dem Wasserbad beim Erkalten an der Oberfläche eine amorphe Abscheidung, nach deren Zertrïmmerung eine neue auftrat, bis schließlich die ganze Masse fest geworden war.

Eine Baryumbestimmung, welche der Formel $\left(\mathrm{C}_{13} \mathrm{H}_{14} \mathrm{NO}_{5}\right)_{2}$ $\mathrm{Ba}$ entspricht, wurde schon früher mitgeteilt. Eine $15 \%$ ige wässerige Lösung drehte $0,4^{0}$ rechts.

Kaliumsalz. Es wurde durch Umsetzen des Baryumsalzes mit Kaliumsulfat dargestellt. Aus der stark eingeengten wässerigen Lösung schieden sich auf Zusatz von Alkohol wohl Krystalle ab, aber es gelang bisher nicht, sie in für die Analyse genügender Menge zu erhalten. Eine 15\% ige wässerige Lösung drehte $0,8^{\circ}$ nach rechts.

Brucinsalz. Es ist das einzige Salz, das wir bisher schön krystallisiert erhalten haben. Es entsteht beim Erhitzen der wässerigen Lösung der freien Säure mit der berechneten Menge (2 Mol.) Brucin, scheidet sich beim Erkalten ab und läßt sich aus heißem Wasser umkrystallisieren. Für die Analyse wurde es bei $80^{\circ}$ im Vakuum getrocknet.

0,1065 g Substanz: 0,2629 g CO$_{2}, 0,0632 \mathrm{~g} \mathrm{H}_{2} \mathrm{O}$

- 0,1697 g Substanz: 10,4 ccm N (170, $738 \mathrm{~mm})$ $\mathrm{C}_{13} \mathrm{H}_{15} \mathrm{NO}_{5} \cdot 2 \mathrm{C}_{23} \mathrm{H}_{26} \mathrm{~N}_{2} \mathrm{O}_{4}$ ber. C $67,20 \mathrm{H} \mathrm{6,41} \mathrm{N} \mathrm{6,65}$ gef. , $67,32,6,64,6,87$.

Eine 2,7\% ige Lösung in 30\% igem Alkohol drehte 1,370 nach links.

Synthese der Phenylacetylglutaminsäure. Je $1 \mathrm{~g}$ d-Glutaminsäure wurden mit $20 \mathrm{ccm}$ Wasser und $5 \mathrm{~g}$ Natriumbicarbonat unter allmählichem Zusatz von $3 \mathrm{~g}$ Phenylacetylchlorid geschüttelt, bis der Geruch nach dem Chlorid verschwunden war. Nach dem Absaugen und Ansäuern mit Phosphorsäure wurde mit Äther und darauf mit Essigäther ausgeschüttelt. Die Rückstände, welche nach dem Verdunsten des Essigäthers hinterblieben, krystallisierten, die Krystalle waren 
aber sehr hygroskopisch. Sie wurden vereinigt und wieder in Essigäther gelöst. Die Lösung, mit dem gleichen Volumen Äther versetzt, schied beim Stehen in verschlossenem Kolben allmählich einen gelben Bodensatz $a b$, welcher aus Essigäther und Wasser wie die Phenylacetylglutaminsäure kristallisierte, auch die fast gleiche spezif. Drehung zeigte, aber sehr hygroskopisch war, offenbar infolge einer geringen Beimengung. Aus der überstehenden äthylacetat-ätherischen Lösung aber erhielten wir eine mit der natürlichen Phenylacetylglutaminsäure völlig identische Verbindung. Schmelzpunkt $122-123^{\circ}$.

Analyse. 0,1223 g Substanz: 0,2634 $\mathrm{g} \mathrm{CO}_{2}, 0,0633 \mathrm{~g}$ $\mathrm{H}_{2} \mathrm{O}-0,1315 \mathrm{~g}$. Substanz: $6,55 \mathrm{ccm} \mathrm{N}\left(16^{\circ}, 723 \mathrm{~mm}\right)$.

$$
\begin{aligned}
& \mathrm{C}_{13} \mathrm{H}_{15} \mathrm{NO}_{5} \text { ber. } \mathrm{C} 58,84 \mathrm{H} 5,70 \mathrm{~N} \mathrm{5,28} \\
& \text { gef. „58.74 „5,79 ,5,49. }
\end{aligned}
$$

0,0802 g Substanz erfordern bei der Titration $5,99 \mathrm{ccm}$ $\mathrm{n} / 10$-Lauge (berechnet $6,05 \mathrm{ccm}$ ).

Polarisation. 0,2586 g Substanz. Gesamtgewicht der Lösung 7,5932 g. Spez. Gewicht 1,0093. Prozentgehalt 3,406. Drehung bei $18^{\circ} \mathrm{im} 2 \mathrm{dm}$-Rohr bei Natriumlicht $1,31^{\circ}$ nach links. Also $[\alpha]_{D}^{18^{\circ}}=-19,05^{\circ}$.

\section{Verhalten der Phenylacetylglutaminsäure im menschlichen Körper.}

Der eine von uns (Sh.) nahm 4,5 g der Säure als Natriumsalz in wässeriger Lösung per os. Er verzehrte in den der Einnahme vorangehenden 16 Stunden und den auf sie folgenden 24 Stunden nur kleine Mengen von Brot mit Butter und Kakao. Der. während 24 Stunden nach der Zufuhr gebildete Harn wurde in 4 Portionen gesammelt, gemessen und polarisiert.

\begin{tabular}{|c|c|c|c|c|c|}
\hline & (5 St.) & 325 & $"$ & "פ & $0,14^{\circ}$ \\
\hline & (5 St.) & 150 & & & $0,09^{\circ}$ \\
\hline & (7 St.) & 470 & " & " & $0,07^{\circ}$ \\
\hline
\end{tabular}

1. Portion (7 St.) $1150 \mathrm{ccm}$ drehte $0,12^{\circ}$ links

Die Polarisation der vierten Portion ergab denselben Wert, wie die der unmittelbar vor der Einnabme der Säure gelassenen. $\mathrm{DaB}$ die Ausscheidung nach 24 Stunden beendet ist, hatte auch 
schon ein früherer Versuch, in dem Phenylacetylglutamin ${ }^{1}$ ) eingeführt worden war, ergeben.

Die gesamte Harnmenge wurde bei neutraler Reaktion und mäßiger Temperatur eingedampft und nach Ansäuern mit Phosphorsäure dreimal (im ganzen 11 Stunden) mit Äthylacetat extrahiert. Die beiden ersten Extrakte blieben auch bei längerem Stehen ganz klar und schieden keine Krystalle ab. Da schon nach Eingabe von $1 \mathrm{~g}$ Phenylessigsäure aus den ersten entsprechend gewonnenen Essigätherextrakten beim Stehen Phenylacetylglutamin auskrystallisiert, so sprach das Ausbleiben einer Krystallabscheidung gegen die Umwandlung der eingegebenen Glutaminsäureverbindung in die Glutaminverbindung.

Wir verdunsteten jetzt die vereinigten drei Essigätherextrakte, versetzten den Rückstand mit Barytwasser bis zur alkalischen Reaktion, behandelten mit Kohlensäure, filtrierten, dampften zur Trockene ein und kochten mehrmals mit abs. Alkohol aus, um den Harnstoff zu entfernen. Jetzt wurde mit Wasser aufgenommen, die wässerige Lösung mit Phosphorsäure angesäuert und mit Essigäther extrahiert. Der Essigätherrückstand krystallisierte auf Zusatz von Äther völlig und die Menge betrug $3 \mathrm{~g}$. Der Schmelzpunkt lag bei $105^{\circ}$. Bei der Titration verbrauchten $0,1594 \mathrm{~g} 10,47 \mathrm{ccm}$ n/10-Lauge statt der für Phenylacetylglutaminsäure berechneten $12,02 \mathrm{ccm}$. Die Verunreinigung erwies sich im wesentlichen als Hippursäure. Beim Erkalten der heißen Essigätherlösung krystallisierte diese Säure in reinem Zustande aus. Sie wurde abfiltriert und das Filtrat fraktioniert mit Äther gefällt. Schmelzpunkt und spez. Drehung dieser Fraktionen lagen den für Phenylacetylglutaminsäure gefundenen sehr nahe. Sie wurden deshalb vereinigt und mit Essigäther in der Wärme behandelt. Dabei blieb eine kleine Menge ungelöst und wurde durch Filtration entfernt. Das Filtrat wurde verdunstet, der Rückstand wurde mit Essigäther erhitzt, das Ungelöste abfiltriert und dieses Verfahren mehr-

1) Phenylacetylglutamin wird unverändert vom Menschen wieder ausgeschieden. Von 5,52 $\mathrm{g}$ eingeführter Substanz gewannen wir aus dem 24 stündigen Harn 3,18 g, d. h. 57,6\% in reinem Zustande wieder. 0,1693 g verbrauchten bei der Titration $6,38 \mathrm{ccm}$ n/10-Lauge (berechnet $6,41 \mathrm{ccm}$ ). 
mals widerholt. Nachdem so die in heißem Äthylacetat schwerlöslichen und unlöslichen Beimengungen entfernt worden waren, erfolgte die Krystallisation in der für die Phenylacetylglutaminsäure charakteristischen Weise (Herauswachsen blumenkohlartiger Massen über die Oberfläche). Auch die weitere Untersuchung ergab, daß es sich um diese Säure handelte. Schmelzpunkt $122-122,5^{\circ}$.

Analyse. 0,1492 g Substanz: 7,25 ccm N $\left(19,5^{\circ}, 743 \mathrm{~mm}\right)$. $\mathrm{C}_{13} \mathrm{H}_{15} \mathrm{NO}_{5}$ ber. $\mathrm{N} 5,28$

0,1004 g Subst. verbrauchten 7,51 $\mathrm{ccm}^{\mathrm{n}} /_{10}$-Lauge (ber. 7,60 ccm).

Spez. Drehung. 0,3393 g Substanz. Gesamtgewicht der Lösung 8,1314 g. Spez. Gewicht 1,0112. Prozentgehalt 4,1727. Drehung bei $18^{\circ}$ im $2 \mathrm{dn}-$ Rohr bei Natriumlicht $1,61^{\circ}$ nach links. Also $[\alpha]_{D}^{18^{\circ}}=-19,08^{\circ}$. 